\title{
ARTICLE
}

\section{Buprenorphine prescription, misuse and service provision: a global perspective}

\author{
Roger Chun Man Ho, Kai Yang Chen, Birit Broekman \& Anselm Mak
}

Roger Chun Man Ho is a psychiatric specialist registrar at the National University of Singapore. He has a special interest in psychoneuroimmunology and addiction. Kai Yang Chen is a research assistant in the Faculty of Medicine, Dentistry and Health Science at the University of Western Australia. His current research interests are substance misuse and psychological trauma. He has also gained experience working at the Sexual Assault Referral Centre in Perth, Australia. Birit Broekman is a psychiatrist in the Department of Child and Adolescent Psychiatry, Academic Medical Centre, De Bascule, University of Amsterdam, The Netherlands. Her special interests include post-traumatic stress disorder and substance misuse in adolescents. Anselm

Mak is Assistant Professor of

Medicine and Rheumatology at Yong Loo Lin School of Medicine, National University of Singapore. He has a special interest in immunological complications associated with substance misuse.

Correspondence DrRoger Ho, Department of Psychological Medicine, Level 5, National University Hospital, 5 Lower Kent Ridge Road, Singapore 119074. Email: pcmrhcm@nus.edu.sg

\begin{abstract}
SUMMARY
Buprenorphine, a partial $\mu$-opioid agonist and $\kappa$-opioid antagonist, is recommended as safe and effective maintenance treatment for opioid dependence. It offers the possibility of management in primary care settings. However, its prescription has led to diversion for illicit recreational use and resulted in medical complications and, rarely, fatal overdose in combination with other sedatives. The outcome of buprenorphine maintenance programmes varies from country to country and it is determined by the local therapeutic traditions, regulatory restrictions and existing service provision for opioid misusers. This article addresses the pharmacology of buprenorphine, the benefits and drawbacks of its prescription, service provision for opioid misuse around the world, policy recommendations, and prescribing training requirements.
\end{abstract}

\section{DECLARATION OF INTEREST}

None.

Illicit opioid use is increasing worldwide and heroin is a robust reinforcer in misuse liability, leading to self-administration (Comer 2002). According to the European Monitoring Centre for Drugs and Drug Addiction (2007), the prevalence of opioid use in Europe is between 1 and 6 individuals per 1000 adult population (aged between 15 and 64 years). In 2005, more than 585000 opioid users received substitution treatment in Europe. In the UK, 1\% of the population between 15 and 24 years of age were estimated to be chronic illicit drug users. Of the 160000 people who sought help relating to their drug use between 2004 and 2005, 75\% reported an opioid as their main problem drug. Participation in opioid agonist treatment ranged from 50 to $80 \%$ in the UK.

One of the primary objectives of treatment for opioid misuse is to offer substitution therapy with a long-acting agonist for either maintenance or detoxification. Detoxification generally refers to techniques that relieve and foreshorten the withdrawal syndrome, and it should be readily available for patients who have expressed an informed choice to become abstinent. The National Institute for Health and Clinical Excellence (NICE) (2007) has described five basic principles of opioid detoxification (Box 1). The effectiveness of detoxification can be measured in terms of symptom reduction on the Objective Opiate Withdrawal Scale (Handelsman 1987). Maintenance therapy refers to the long-term treatment of patients who want to stop illicit drug use but are unable to achieve abstinence.

In opioid dependence, there are three major approaches: substitution therapy, abstinenceoriented pharmacotherapy and psychological interventions (Hulse 2002). An effective maintenance treatment programme should demonstrate retention in treatment, reduction of illicit drug use and continuous abstinence (Mattick 2002). Secondary measures include improvements in quality of life and global functioning. The

B0X 1 The five basic principles of opioid detoxification

- Assess people presenting for detoxification to establish the presence and severity of opioid dependence and use of other substances, including alcohol, benzodiazepines and stimulants. Drug screening tests (e.g. urinalysis) are recommended for all patients before starting substitution therapy

- If opioid dependence or tolerance is uncertain, use confirmatory laboratory tests in addition to 'nearpatient testing' (quick tests not requiring complex instruments), particularly in young patients for first-time detoxification, and in patients with inconsistent clinical assessment and complex patterns of drug misuse

- Give the patient detailed information about detoxification and the associated risks. The treating doctor should discuss the physical and psychological aspects and interventions of opioid withdrawal

- Consider the best pharmacological intervention (e.g. buprenorphine, methadone, lofexidine) for detoxification

- Continue treatment and support after detoxification

(National Institute for Health and Clinical Excellence 2007) 
BOX 2 Recommendations on prescription of opioid maintenance medication

- Medications with a relevant product licence should ordinarily be used (i.e. methadone and buprenorphine)

- Practitioners should be willing to prescribe high daily doses of maintenance treatment to achieve optimal benefit (methadone: daily dose between 60 and $120 \mathrm{mg}$; buprenorphine: daily dose between 16 and $32 \mathrm{mg}$ )

- There should be greater use of arrangements for daily dispensing (to increase adherence and reduce the risk of diversion to the illicit market)

- Arrangements for supervised consumption of daily doses should be introduced (to increase adherence and reduce risk of diversion)

- Doctors should not prescribe 'take-home' methadone tablets (as these can be crushed and injected)

- Doctors outside specialist centres should not ordinarily prescribe 'take-home' injectable methadone ampoules

- General practitioners should be involved increasingly in the provision of such treatment

(Department of Health 1999)

Department of Health (1999) and NICE (2007) made a number of clear recommendations regarding the prescription of opioid maintenance medication (Boxes $2 \& 3$ ).

\section{The controversy in buprenorphine maintenance}

Buprenorphine, a semi-synthetic partial $\mu$-opioid agonist and $\kappa$-opioid antagonist, is advocated as safe and effective maintenance treatment for opioid dependence (Lapeyre-Mestre 2003; Auriacombe 2004; Magura 2007). It has been approved for treating opioid dependence in the UK since 1999.

\section{BOX 3 Recommendations on the choice of medication for opioid detoxification}

- Offer either methadone or buprenorphine as first-line treatment:

normally start detoxification with the same medication as planned for any maintenance treatment

consider the preference of the service user

- Lofexidine may be considered for people:

who have made an informed and clinically appropriate decision (not to use methadone or buprenorphine or detoxify within a short time period)

with mild or uncertain dependence (including young people)

- Do not routinely use clonidine

(National Institute for Health and Clinical Excellence 2007)
Defined in the Misuse of Drugs Act 1971 as a Class C drug, buprenorphine falls within Schedule 3 of the Misuse of Drugs Regulations 2001. The British Association for Psychopharmacology (LingfordHughes 2004) recommends buprenorphine for rapid withdrawal of opioids and there is emerging evidence to support its use in pregnancy (Box 4). Mattick et al (2002) argued that morbidity and mortality associated with heroin dependence could be reduced with buprenorphine. Luty et al (2005) proposed that buprenorphine should replace methadone as a treatment for heroin dependence. However, it is unclear whether this recommendation is justified, as there is evidence of diversion (use for recreational purposes) and misuse of prescribed buprenorphine (Jenkinson 2005). Adverse events have emerged, including local cutaneous complications after injection (Ho 2009) and fatal overdoses associated with concomitant benzodiazepine misuse (Tracqui 1998). Furthermore, dihydrocodeine, an oral opioid, is a viable alternative to methadone as maintenance treatment but it has not received much attention outside Europe (Robertson 2006).

In an attempt to address this controversy, we will briefly discuss the pharmacology of buprenorphine, the benefits and drawbacks of prescribing it, the prevalence of its misuse and the drug's potential negative consequences. We will also consider the

BOX 4 Recommendations from the British Association for Psychopharmacology on the management of withdrawal from opioid drugs

There is a substantial evidence base for three main types of pharmacotherapy: methadone, buprenorphine and $\alpha_{2}$-adrenergic agonists (e.g. clonidine and lofexidine). All are effective in reducing withdrawal symptoms. The choice of agent may be guided by the following.

Desired duration of treatment

- If short duration of treatment is desirable, $\alpha_{2}$-adrenergic agonists are preferable to methadone

- Buprenorphine can be used for rapid withdrawal of opioids and has a better outcome than clonidine

- Methadone treatment is more successful if it follows a slower, linear dose reduction regimen, which will result in fewer craving or withdrawal symptoms and enhance completion rates of detoxification

\section{Adverse effects}

- Buprenorphine is preferable to $\alpha_{2}$-adrenergic agonists if there are concerns about bradycardia or hypotension

Withdrawal severity

- Buprenorphine results in lower severity of withdrawal symptoms than $\alpha_{2}$-adrenergic agonists

Specific patient groups

- Methadone can be used during pregnancy, and there are emerging studies regarding the use of buprenorphine. The $\alpha_{2}$-adrenergic agonists should not be prescribed in pregnancy

(Lingford-Hughes 2004) 
service provision of buprenorphine from a global perspective, and suggest policy recommendations and training requirements.

\section{Some facts about buprenorphine}

\section{History and formulations}

Buprenorphine, an oripavine derivative of the opium alkaloid thebaine, was first developed in a search for opioid compounds with mixed agonistantagonist effects (Lewis 1985). As a parenteral analgesic it is $25-40$ times more potent than morphine (Houde 1979). It was shown to relieve acute opioid withdrawal by Jasinski et al (1989). Currently available preparations are buprenorphine alone and the combination of buprenorphine and the antagonist naloxone (buprenorphine/naloxone). Buprenorphine is available in two formulations (low and high dose) in the UK. The low-dose formulation (licensed for the treatment of moderate to severe pain) is available both in sublingual tablet (0.2 and $0.4 \mathrm{mg})$ and injection forms $(0.3 \mathrm{mg})$. The high-dose formulation, which became available in the UK in 1999 and is licensed for the treatment of opioid addiction, is available as $0.4,2$ and $8 \mathrm{mg}$ sublingual tablets (Schifano 2005).

Buprenorphine is easily soluble and it can be dissolved and illicitly injected (Ford 2004).

\section{B0X 5 General principles of prescribing buprenorphine}

- Doctors prescribing buprenorphine medication should ensure that they have the most upto-date information available. Balanced and regularly updated information can be obtained from the British Association for Psychopharmacology and NICE.

- Before buprenorphine is prescribed, the psychosocial factors associated with opioid dependence and the options of psychological treatment should be carefully explained to the patient.

- Before prescribing buprenorphine, the doctor should clearly document the reasons why the medication is being prescribed and that the risks and benefits have been discussed.

- Adequate single daily dose should be used and polypharmacy should be avoided. Monitor the patient closely. Continue with full documentation of its effectiveness.

- Addiction specialists should always work in partnership with patients, their family and GPs, and be able to discuss the risks and benefits of prescribing buprenorphine. It is important to achieve a balance between patient autonomy and structure in the treatment programme.

- Addiction specialists should identify patients who are stabilised on buprenorphine with low risk of misuse and refer them to general practice-based or office-based treatment in the community. General practitioners can continue to oversee the general well-being of patients with opioid dependency.

- Treatment should include behavioural therapy based on close monitoring of illicit drug use by supervised urine sample collection, reinforcement of treatment adherence and relapse prevention group therapy.

- Psychiatrists, accident and emergency department physicians and GPs should be aware of the potential intravenous misuse of buprenorphine. If diversion is detected, they should consider withdrawing buprenorphine. The type of diversion and reasons for withdrawal should be documented and alternative treatments considered.

\section{Pharmacology and dosage}

Buprenorphine is metabolised in the body by both $\mathrm{N}$-dealkylation and conjugation by the cytochrome P450 enzyme system, and excreted principally in faeces and urine. Peak plasma concentration occurs approximately $3 \mathrm{~h}$ after dose administration (Jasinski 1989) and the drug has a terminal half-life of about 3-5h. The titration dosing for induction starts with 2 or $4 \mathrm{mg}$ on the first day. The dose can then be increased in 2-4 mg increments to $12-16 \mathrm{mg}$ on the second day. Most patients can be stabilised on 8-32 mg/day.

Comer et al (2001) found that a group of intravenous heroin users who were receiving $16 \mathrm{mg}$ buprenorphine a day reported a greater reduction in self-administration of heroin than a group on $8 \mathrm{mg}$ buprenorphine. As a maintenance dose, 12$16 \mathrm{mg}$ buprenorphine is as effective as $50-80 \mathrm{mg}$ methadone (Gowing 2004).

A study of buprenorphine/naloxone combination reported that $8 \mathrm{mg}$ buprenorphine/ $2 \mathrm{mg}$ naloxone and $32 \mathrm{mg}$ buprenorphine/8 $\mathrm{mg}$ naloxone were well tolerated and effective in reducing the reinforcing effects of heroin, relative to a dose of $2 \mathrm{mg}$ buprenorphine/0.5 mg naloxone (Comer 2005).

\section{Side-effects}

The primary side-effects of buprenorphine are similar to those of other $\mu$-opioid agonists: nausea, vomiting and constipation. These side-effects may be less intense than those produced by full agonist opioids. Hypersensitivity to buprenorphine is rare (fewer than $1 \%$ of patients). Nevertheless, buprenorphine is absolutely contraindicated if the patient has a history of hypersensitivity to it and relatively contraindicated if the patient is consuming alcohol or taking sedative hypnotics such as benzodiazepines or barbiturates. The general principles of prescribing buprenorphine are summarised in Box 5.

\section{Pros and cons of buprenorphine prescription}

\section{Arguments in favour}

In theory, the pharmacodynamic properties of buprenorphine make it an ideal drug for maintenance treatment in opioid dependence, and its low potential for overdose and misuse make it particularly suitable for out-patient treatment. Plasma concentrations of the drug increase linearly with increasing dose until a plateau is reached. The partial agonistic property of buprenorphine reduces the likelihood and severity of overdose, and limits liability to physical dependence (Lewis 1985; Jasinski 1989). 
In naturalistic primary care practice, where dual diagnosis is common, Magura et al (2007) reported that two-thirds of patients remained on buprenorphine treatment. Patients living in their own home or those misusing prescription opioids (rather than heroin) were more likely to be retained. Pharmacists have expressed positive attitudes towards buprenorphine and most were no more concerned about prescription forgery and diversion of this drug than of any other narcotic medication (Raisch 2003).

At high doses, buprenorphine exhibits narcotic antagonist activity (Dum 1981), which also helps limit possible misuse. Buprenorphine is safe at high intravenous doses (Comer 2005), producing few changes in arterial oxygen saturation (Walsh 1994). High buprenorphine doses have been shown to suppress self-administration of other drugs of misuse such as alcohol (Ciccocioppo 2007). Auriacombe and colleagues (2004) reviewed the use of buprenorphine during pregnancy and found that neonatal opioid withdrawal with buprenorphine was less severe and of shorter duration than that with methadone; however, its UK licence does not cover breastfeeding mothers (Ford 2004).

\section{Arguments against}

It should be noted that the low misuse potential of buprenorphine has been based on animal studies (Dum 1981) and its partial agonist role is often overplayed. In humans, illicit intravenous use of the drug can produce euphoria and opioid-like effects (Elkader 2005); the potential for illicit use is similar to that for morphine or hydromorphone in heroin users, recently detoxified opiate misusers and non-opioid-dependent volunteers (Zacny 1997; Comer 2002). This may undermine the self-administration strategies associated with buprenorphine maintenance therapy. Sublingual preparations of buprenorphine are often injected intravenously by patients (Ho 2009).

Buprenorphine use can lead to undesired withdrawal effects due to its higher affinity than methadone for opiate receptors (Clark 2002). Withdrawal was observed in opioid-dependent laboratory animals treated with buprenorphine (Yanagita 1982), and intravenously administered buprenorphine served as a reinforcer in both human and non-human primates (Comer 2005). One potential variable that contributed to the pattern of buprenorphine self-injection was the presence of withdrawal symptoms.

Intravenous buprenorphine misuse may lead to cutaneous complications such as abscesses and it may also increase the risk of infectious hepatitis and HIV infection (Auriacombe 2004). Despite the potential for harmful effects, studies looking into the prevalence and reasons for intravenous misuse are scant. In a recent study, we reported the frequency of medical complications among 130 intravenous buprenorphine misusers (97\% males, $3 \%$ females) admitted to the National University Hospital, Singapore, who also tended to use other drugs, particularly midazolam (70\%) (Ho 2009). The frequency of medical complications was as follows: hepatitis C, $56 \%$; cutaneous complications, $31 \%$; and infective endocarditis, $11 \%$.

Deaths related to heroin/morphine and methadone are common in the UK, although deaths related to buprenorphine are much rarer (International Centre for Drug Policy 2007).

\section{Current evidence and cost-effectiveness of buprenorphine in opioid maintenance treatment}

There have been several controlled trials of buprenorphine replacement therapy for patients with opioid dependency (Table 1). One of the

TABLE 1 Studies of buprenorphine replacement therapy

\begin{tabular}{|c|c|c|c|c|}
\hline Study & $\begin{array}{l}\text { Type of trial } \\
\text { (sample size) }\end{array}$ & Active treatment & Control & Outcomes \\
\hline Comer (2001) & $\begin{array}{l}\text { 6-week, double-blind, } \\
\text { placebo-controlled } \\
\text { in-patient study }(n=8)\end{array}$ & $\begin{array}{l}16 \text { mg sublingual } \\
\text { buprenorphine }\end{array}$ & $\begin{array}{l}8 \mathrm{mg} \text { sublingual } \\
\text { buprenorphine }\end{array}$ & $16 \mathrm{mg}$ buprenorphine reduced heroin self-administration relative to $8 \mathrm{mg}$ \\
\hline $\begin{array}{l}\text { Lapeyre- } \\
\text { Mestre (2003) }\end{array}$ & $\begin{array}{l}\text { 24-week } \\
\text { observational cohort } \\
\text { study }(n=282)\end{array}$ & $\begin{array}{l}\text { Buprenorphine } \\
\text { treatment }\end{array}$ & Nil & $\begin{array}{l}50 \% \text { rational users (no more than two prescriptions); } 24 \% \text { occasional } \\
\text { users (less than two buprenorphine prescriptions); } 26 \% \text { chaotic drug } \\
\text { users (three or more prescriptions). The overall } 24 \text {-week treatment } \\
\text { retention rate was } 37 \%\end{array}$ \\
\hline Comer (2005) & $\begin{array}{l}\text { 3-week double-blind, } \\
\text { placebo-controlled } \\
\text { in-patient study }(n=6)\end{array}$ & $\begin{array}{l}\text { Intravenous } \\
\text { buprenorphine }\end{array}$ & $\begin{array}{l}\text { Intravenous } \\
\text { methadone }\end{array}$ & $\begin{array}{l}\text { The ratings of 'good drug effect', 'liking' and 'high' increased after } \\
\text { administration of buprenorphine and methadone; the two drugs were } \\
\text { equally effective in producing reinforcing and subjective effects under } \\
\text { experimental conditions }\end{array}$ \\
\hline Kakko (2007) & $\begin{array}{l}\text { 6-month double- } \\
\text { blind randomised } \\
\text { controlled trial }(n=96)\end{array}$ & $\begin{array}{l}\text { Stepped treatment } \\
\text { with buprenorphine/ } \\
\text { naloxone }\end{array}$ & Methadone & $\begin{array}{l}\text { 6-month retention was } 78 \% \text {. Proportion of urine samples free of illicit } \\
\text { opiates reached } 80 \% \text { in both arms over time. Stepped treatment and } \\
\text { methadone maintenance therapy outcomes were virtually identical }\end{array}$ \\
\hline
\end{tabular}


BOX 6 Costs of buprenorphine, methadone and dihydrocodeine

Buprenorphine

- $2 \mathrm{mg}$, 50-tablet pack = $£ 5.33$

- $4 \mathrm{mg}, 50$-tablet pack $=£ 10.66$

Methadone

- $10 \mathrm{mg} / \mathrm{ml}=£ 12.01$

- $20 \mathrm{mg} / \mathrm{ml}=£ 24.02$

Dihydrocodeine tartrate

- $40 \mathrm{mg}$, 100-tablet pack = £11.51

(British Medical Association 2008)

largest randomised controlled trials involved 96 individuals, who were randomised either to stepped treatment with buprenorphine/naloxone in combination or to methadone. Nearly $80 \%$ reported abstinence during the 6 -month treatment period in both treatment groups, with identical outcomes (Kakko 2007). A trial comparing intravenous buprenorphine with intravenous methadone found that both treatments were equally effective in producing reinforcing and subjective effects under experimental conditions (Comer 2005).

The costs of buprenorphine, methadone and dihydrocodeine in the UK are summarised in Box 6. In a US study, Rosenheck \& Kosten (2001) constructed a hypothetical clinical cost scenario comparing the buprenorphine/naloxone combination with methadone. The monthly direct costs of buprenorphine/naloxone were estimated to be US\$580-1900 greater, although the indirect costs were estimated to be lower because of fewer regulatory requirements. In real practice in Australia, Winstock et al (2007) did not find the expected indirect medical cost saving, as the provision of buprenorphine/naloxone requires additional labour in pharmacies. In another Australian study, Doran and colleagues (2003) compared the cost-effectiveness of buprenorphine and methadone, and the difference was not found to be statistically significant. Further evaluation of cost-effectiveness is needed as buprenorphine is likely to come off patent soon and to become available in a much cheaper generic form; buprenorphine/naloxone will remain on patent and will therefore be relatively more expensive over the next few years.

\section{Global trends in buprenorphine prescribing England}

The Department of Health (1999) has recommended specialist initiation of buprenorphine treatment with long-term supervised dispensing to prevent intravenous misuse. De Wet et al (2005) reported that buprenorphine prescription rates increased disproportionately to those for methadone in 28 strategic health authorities in England. By the end of 2003, the number of buprenorphine prescriptions had increased to $23 \%$, accounting for $45 \%$ of opioid prescription costs. Strang and colleagues (2007) studied the change in prescription practice by general practitioners (GPs) in the management of opioid users from 1995 to 2005 and concluded that the number of substitute opioid prescriptions had doubled in the UK. Methadone was the most commonly prescribed, but its use fell from 97\% in 1995 to $83 \%$ in 2005. Conversely, the share of buprenorphine increased from $10 \%$ in 1995 to $16 \%$ in 2005 . In general practice, $47 \%$ of doctors prescribed buprenorphine on a daily basis (patients had to go to the surgery each day to collect their prescription), 21\% prescribed several times per week (patients received a 2- to 3-day supply on a single prescription) and $32 \%$ on a weekly basis (a 7-day supply on a single prescription). The pattern is similar in hospital settings: $49 \%$ prescribed buprenorphine on a daily basis, $23 \%$ prescribed several times per week and $28 \%$ on a weekly basis.

In 2007, the National Programme on Substance Abuse Deaths reported 217 deaths related to methadone use in the UK. As a proportion of all substance-related deaths, methadone-related deaths had increased from $12 \%$ in 2006 to $17 \%$ in 2007 (International Centre for Drug Policy 2007). Between 1980 and 2002, buprenorphine was mentioned in 43 fatalities, 14 (33\%) of which occurred after 1999, when high-dose formulations of buprenorphine entered the UK market (Schifano 2005). It was found that the deceased had often misused buprenorphine in combination with benzodiazepines and other opioids.

\section{Ireland and Scotland}

Six years after buprenorphine was introduced as an analgesic in Ireland, a study of opioid users presenting for treatment at one centre found that the proportion who reported buprenorphine misuse jumped steeply from 0 to $80 \%$ in a 12-month period (O’Connor 1988).

In Scotland, buprenorphine had become the most common drug of intravenous misuse by the early 1990s, resulting in its withdrawal from the market, although it was reintroduced in 2003 (Jenkinson 2005). A national questionnaire survey commissioned by the Scottish Drug Specialist Committee in 2003 found that about $50 \%$ of clinicians were prescribing the drug; the other $50 \%$ were eager to 
prescribe but felt restrained by the lack of local policies and protocols (Taikato 2005).

\section{France}

Methadone and buprenorphine have been available for opioid maintenance in France since 1996. Conventional methadone maintenance treatment is subject to strict regulations, but buprenorphine follows general freedom of prescription and can be dispensed by any community pharmacist for a maximum of 7 days. Consequently, about 65000 patients a year are treated with buprenorphine about ten times more than with methadone. This has resulted in a substantial reduction (79\%) in deaths by opioid overdose and in the incidence of neonatal opioid withdrawal (Auriacombe 2004). Although the majority (64\%) of regular users of buprenorphine are regarded as 'rational drug users' (receiving the drug from no more than two different prescribers and dispensed by no more than two pharmacists during the study period), $26 \%$ have been identified as 'non-rational (chaotic) drug users' (receiving the drug from three or more prescribers, having irregular access to healthcare, frequently using other psychoactive drugs such as zolpidem and falsifying prescriptions) (LapeyreMestre 2003). 'Doctor-shopping' is an important problem for buprenorphine maintenance treatment in France (Pradel 2004).

Half of the patients prescribed buprenorphine illicitly inject the drug, and both intravenous injection of crushed tablets and a concomitant intake of benzodiazepines have been identified as major risk factors for buprenorphine-related fatalities in France (Kintz 2001). Despite these adverse outcomes, Auriacombe et al (2004) argued that regular misusers of intravenous buprenorphine appear to have better outcomes than those who use it less regularly and those who inject methadone.

\section{The Netherlands}

Dutch addiction care has always been focused on opioid addiction, which is usually treated with methadone. The Dutch addiction care system adopts a 'low-threshold' approach when prescribing methadone. In The Netherlands, buprenorphine is registered as an analgesic and is mainly used for post-operative pain; an import licence is needed from the National Healthcare Inspectorate for importing high-dose buprenorphine. A rapid, large-scale change in prescription practice from methadone to buprenorphine would be unlikely as the national Central Register of Substances has not approved buprenorphine for the treatment of opioid dependence.

\section{The USA}

The US Food and Drug Administration simultaneously approved both buprenorphine alone and the combination of buprenorphine/ naloxone in October 2002. It was reported that over $90 \%$ of buprenorphine prescriptions are for combined buprenorphine/naloxone, owing to the lower potential for misuse (Chua 2006). The Drug Addiction Treatment Act of 2000 requires physicians who prescribe buprenorphine to possess certification in addiction medicine/ psychiatry or to complete $8 \mathrm{~h}$ of training on the treatment of patients with opioid addiction (Cunningham 2006). Each physician is limited to treating 30 patients at a time with the drug, to prevent 'doctor shopping'. The likelihood of misuse of buprenorphine by individuals with heroin dependence is relatively low in the USA (Comer 2007). Buprenorphine, not methadone, is approved for use in primary care settings (Kakko 2007) and a substantial proportion of New York City physicians would prescribe buprenorphine for heroin dependence (Coffin 2006).

\section{Australia}

Buprenorphine was first registered in Australia in 2000. Prescription of high-dose buprenorphine became common and by 2005 about 36\% of individuals on opioid substitution treatment in Victoria were receiving the drug (Jenkinson 2005). In Melbourne, 37\% of patients with opioid dependence reported that they had illicitly injected buprenorphine at some time. This led to the conclusion that effective countermeasures were needed to address diversion and injection of buprenorphine. Theintroduction of buprenorphine/ naloxone to Australia in April 2006 permitted the revision of 'take-home' policies in many states and introduced the possibility of unsupervised treatment (Winstock 2007).

\section{Singapore}

Methadone is not widely prescribed in Singapore, so the launch of buprenorphine in the country in 2002 offered hope, as it was thought that this new drug would have lower misuse potential, owing to its partial agonist profile. However, buprenorphine use became illegal in 2006 because widespread intravenous misuse and associated medical complications had become a substantial healthcare burden for the city-state (Ho 2009). As in the UK in the past (Blackwell 1988), prescribing by GPs was a major source of this opioid diversion. Concomitant benzodiazepine use was common, most often with midazolam (Ho 2009). 
B0X 7 Six strategies for reducing buprenorphine misuse

- Limiting prescription access

- Tailored prescription

- Educating the public

- Educating the professionals

- Prevention of misuse

- Prescription of buprenorphine/naloxone

\section{Recommendations for service provision for prescribing buprenorphine}

The rate of illicit buprenorphine use shows the potential to increase in direct proportion to the prescription rate and general availability. Six strategies of policy change are recommended to reduce buprenorphine misuse (Box 7).

\section{Limiting prescription access}

Limiting access to the prescription of buprenorphine is very important. Those who prescribe the drug should be registered in a nationwide monitoring system to ensure that they are not serving as suppliers to others. The increasing emphasis on risk management and evidence-based practice in the UK might encourage healthcare organisations to monitor duplicate prescriptions, which will minimise lax prescription and deter 'doctor shopping'. Daily supervised dispensing is recommended.

\section{Tailored prescription}

Prescription of buprenorphine and its alternatives should be based on individual patients' characteristics and local guidelines. Buprenorphine and methadone are not mutually exclusive in opioid replacement programmes. Kakko et al (2007) proposed an adaptive stepped-care strategy involving the two drugs that may achieve an optimal balance between safety and efficacy. The patient initially receives up to $32 \mathrm{mg}$ /day buprenorphine. If the clinical effect remains insufficient, the patient can be switched from buprenorphine to methadone. This stepped approach offers the advantage that all patients who need methadone ultimately receive it, but those who do not need it can be successfully treated without it.

It would be desirable to increase patient choice by considering dihydrocodeine (Robertson 2006) but more studies are required to assess patients' preference for this treatment outside Europe.

\section{Educating the public}

Policy should be aimed at warning users as well as patients' families and the public about the negative consequences of buprenorphine misuse. Manufacturers should provide a wide array of more visible platforms (e.g. on labels, posters and advertisements) to discourage the illicit use and unauthorised sales of the drug.

\section{Educating the professionals}

Perhaps the most important recommendation is the development of policies aiming at continuing professional development (continuing medical education). Healthcare workers outside addiction or mental health services seldom find themselves in situations where prevention of buprenorphine misuse is high on their agenda. Education might include the introduction of guidelines formalised by local health authorities, including requirements for additional education for both addiction specialists and GPs to learn about opioid replacement therapy and the potential for diversion of buprenorphine.

\section{Prevention of misuse}

Relapse prevention strategies and cognitivebehavioural approaches may help patients cope with high-risk situations (Marlatt 1985). For example, the patient might identify triggers for craving buprenorphine, promise themselves never to inject it, develop social skills and alternative pleasurable activities, and have a personal emergency plan to deal with relapse, whereas the healthcare provider might give out information sheets documenting the proper use of buprenorphine and the risks associated with intravenous misuse.

\section{Prescription of buprenorphine/naloxone}

Combination buprenorphine/naloxone rather than buprenorphine alone should be promoted as the main medication because the effects of the opioid antagonist naloxone predominate when injected illicitly. Buprenorphine/naloxone has a relatively low street value compared with all other forms of prescription opiates. In recently detoxified heroin misusers, the misuse liability of buprenorphine alone and of buprenorphine/naloxone appear to be similar (Comer 2002).

\section{Training for doctors and pharmacists}

Coffin and colleagues (2006) reported that greater willingness of physicians in New York City to prescribe buprenorphine was associated with more years of licensure, working in a hospital or clinic as opposed to a primary care setting, and being the director of a clinic or programme. In another US study, of online and in-person training in the use of the drug, physicians hesitant to prescribe buprenorphine cited their lack of experience as the main barrier (41\%); a further $24 \%$ were 
concerned about difficulty with dose escalation and stabilisation, and about reimbursement from medical insurance companies (Gunderson 2006). The study suggests that physician training should include in-person training and the availability of consultation after training. Onsite counsellors to offer patients psychoeducation and more information on a drug were also valued, and telephone access to experienced providers significantly improved physicians' confidence (Cunningham 2006; Gunderson 2006).

Allowing trained GPs to prescribe buprenorphine will not only make this 'take-home' maintenance therapy more available, but also offer some form of control and continuity. A study was conducted in Australia to compare pre- and post-training test scores after a training programme for primary care medical practitioners and pharmacists in the delivery of buprenorphine and L- $\alpha$-acetylmethadol treatment in the management of opioid dependence (Lintzeris 2002). Trained doctors and pharmacists were more confident in their therapeutic choices.

\section{Dihydrocodeine: the neglected alternative}

Dihydrocodeine, a semi-synthetic opioid analgesic, has been used in oral formulations for maintenance treatment of opioid dependence in a number of European countries (Macleod 1998). In the UK, dihydrocodeine is a Class B drug available in $30 \mathrm{mg}$ tablets. Among GPs in England and Wales, it is the second most commonly prescribed drug (after methadone) for opiate addiction (Strang 2005). Dihydrocodeine is much shorter acting than methadone because of its lower affinity of binding to the $\mu$-opioid receptor. It is a good alternative for patients who want to avoid methadone, with its sedative effect, dangers of toxicity, and stigma and regulations surrounding prescription and dispensing (Robertson 2006). Common side-effects include constipation, giddiness, hypersensitivity, itching, flushing and other effects of blood vessel dilation. Tolerance, as well as physical and psychological dependence develop with repeated use.

\section{Conclusions}

Detoxification and maintenance treatment for opioid dependence pose particular challenges in maintaining adherence and minimising medication diversion. Before pharmacological treatment is prescribed, strong consideration should be given to psychological therapies such as motivational interviewing and cognitive-behavioural therapy ${ }^{\dagger}$ (McIntosh 2001).

A Cochrane review concluded that buprenorphine is an effective intervention in the maintenance treatment of heroin dependence (Gowing 2004).
Buprenorphine is as effective as methadone at adequate doses and does not significantly differ from methadone in its impact on other substance misuse (Gowing 2004). Global comparison clearly indicates that the preference for methadone or buprenorphine seems primarily due to fashion and regulatory requirements rather than the evidence base. Despite the popularity of buprenorphine prescription in the USA and France, experiences with the drug in Singapore and Scotland suggest that intravenous misuse often occurs. Although there is no single satisfactory explanation to account for the diverse outcomes of buprenorphine in different countries, local therapeutic traditions, regulatory restrictions and existing service provision for opioid misusers are the key determinants of the success of the drug in individual countries. The efficacy of monitoring programmes (of both prescription and misuse) and the social and economic costs of buprenorphine misuse deserve further study.

Diversion and misuse of buprenorphine are likely to be caused by lack of patient supervision, poor training of physicians and loose regulation. Strong warning labels may help, but dispensing buprenorphine must be very carefully regulated and controlled, as this drug has a street value and might therefore be widely disseminated. Monitoring buprenorphine misuse among the high-risk population is important as this group is likely to incur serious medical complications from improper administrations. We also need comparative studies between countries of the extent and principal means of buprenorphine diversion, and of its public and health consequences.

\section{References}

Auriacombe M, Fatseas M, Dubernet J, et al (2004) French field experience with buprenorphine. American Journal on Addictions; 13: S17-28.

Blackwell J (1988) The saboteurs of Britain's opiate policy: overprescribing physicians or American-style 'junkies'? International Journal of Addiction; 23: 517-26.

British Medical Association, Royal Pharmaceutical Society of Great Britain (2008) British National Formulary 55. Pharmaceutical Press.

Chua SM, Lee TS (2006) Abuse of prescription buprenorphine, regulatory controls and the role of the primary physician. Annals of Academic Medicine Singapore; 35: 492-5.

Ciccocioppo R, Economidou D, Rimondini R, et al (2007) Buprenorphine reduces alcohol drinking through activation of the nociceptin/orphanin F0-NOP receptor system. Biological Psychiatry; 61: 4-12.

Clark NC, Lintzeris N, Muhleisen PJ (2002) Severe opiate withdrawal in a heroin user precipitated by a massive buprenorphine dose. Medical Journal of Australia; 176: 167-8.

Coffin PO, Blaney S, Fuller C, et al (2006) Support for buprenorphine and methadone prescription to heroin-dependent patients among New York City physicians. American Journal of Drug and Alcohol Abuse; 32: $1-6$

Comer SD, Collins ED, Fischman MD (2001) Buprenorphine sublingual tablets: effects on IV heroin self-administration by humans. Psychopharmacology; 154: 28-37.
${ }^{\dagger}$ These and other interventions were discussed in Advances by Luty (What works in drug addiction? 2003; 9: 280-7) and Seivewright (Invited commentary on: What works in drug addiction? 2003; 9: 287-8. 


\begin{tabular}{lllll}
\multicolumn{5}{l}{ MCO answers } \\
1 & 2 & 3 & 4 & 5 \\
af & af & at & af & af \\
bf & bt & bf & bf & bt \\
ct & cf & cf & cf & cf \\
df & df & df & dt & df \\
ef & ef & ef & ef & ef
\end{tabular}

Comer SD, Collins ED (2002) Self-administration of intravenous buprenorphine and the buprenorphine/naloxone combination by recently detoxified heroin abusers. Journal of Pharmacology and Experimental Therapeutics; 303: 695-703.

Comer SD, Sullivan MA, Walker EA (2005) Comparison of intravenous buprenorphine and methadone self-administration by recently detoxified heroin-dependent individuals. Journal of Pharmacology and Experimental Therapeutics; 315: 1320-30.

Comer SD, Sullivan MA, Whittington RA, et al (2007) Abuse liability of prescription opioids compared to heroin in morphine-maintained heroin abusers. Neuropsychopharmacology; 1: 1-13.

Cunningham CO, Sohler NL, McCoy K, et al (2006) Attending physicians' and residents' attitudes and beliefs about prescribing buprenorphine at an urban teaching hospital. Family Medicine; 38: 336-40.

Department of Health (1999) Drug Misuse and Dependence. Guidelines on Clinical Management. HMSO

De Wet CJ, Reed LJ, Bearn J (2005) The rise of buprenorphine prescribing in England: analysis of NHS regional data, 2001-03. Addiction; 100: 495-9.

Doran CM, Shanahan M, Mattick RP, et al (2003) Buprenorphine versus methadone maintenance: a cost-effectiveness analysis. Drug and Alcohol Dependence; 71: 295-302.

Dum J, Bläsig J, Herz A (1981) Buprenorphine: demonstration of physical dependence liability. European Journal of Pharmacology; 70: 293-300.

Elkader A, Sproule B (2005) Buprenorphine. Clinical pharmacokinetics in the treatment of opioid dependence. Clinical Pharmacokinetics; 44: $661-80$.

European Monitoring Centre for Drugs and Drug Addiction (2007) Annual Report 2007: The State of the Drugs Problem in Europe. Office for Official Publications of the European Communities (http://www.emcdda.europa. eu/attachements.cfm/att_44705_EN_TDAC07001ENC.pdf).

Ford C, Morton S, Lintzeris N, et al (2004) Guidance for the Use of Buprenorphine for the Treatment of Opioid Dependence in Primary Care. Royal College of General Practitioners (http://www.rcgp.org.uk/PDF/ drug_buprenorphine.pdf).

Gowing L, Ali R, White J (2004) Buprenorphine for the management of opioid withdrawal. Cochrane Database of Systematic Reviews, issue 4: CD002025

Gunderson EW, Fiellin DA, Levin FR, et al (2006) Evaluation of combined online and in person training in the use of buprenorphine. Substance Abuse; 27: 39-45.

Handelsman L, Cochrane KJ, Aronson MJ, et al (1987) Two new rating scales for opiate withdrawal. American Journal of Alcohol Abuse; 13 : 293-308.

Ho RC, Ho EC, Mak A (2009) Cutaneous complications among i.v. buprenorphine users. Journal of Dermatology; 36: 22-9.

Houde RW (1979) Analgesic effectiveness of the narcotic agonistantagonists. British Journal of Clinical Pharmacology; 7: 2975-3085.

Hulse G, White J, Cape G (2002) Management of Alcohol and Drug Problems. Oxford University Press.

International Centre for Drug Policy (2007) Drug-Related Deaths in the UK. Annual Report. St George's Hospital, University of London.

Jasinski DR, Fudala PJ, Johnson RE (1989) Sublingual versus subcutaneous buprenorphine on opiate abusers. Clinical Pharmacology and Therapeutics; 45: 513-9

Jenkinson RA, Clark NC, Fry CL, et al (2005) Buprenorphine diversion and injection in Melbourne, Australia: an emerging issue? Addiction: 100: 197-205.

Kakko J, Grönbladh L, Svanborg KD, et al (2007) A stepped care strategy using buprenorphine and methadone versus conventional methadone maintenance in heroin dependence: a randomised controlled trial. American Journal of Psychiatry; 164: 797-803.

Kintz P (2001) Deaths involving buprenorphine: a compendium of French cases. Forensic Science International; 121: 65-9.

Lapeyre-Mestre M, Llau ME, Gony M, et al (2003) Opiate maintenance with buprenorphine in ambulatory care: a 24-week follow-up study of new users. Drug and Alcohol Dependence; 72: 297-303.
Lewis JW (1985) Buprenorphine. Drug and Alcohol Dependence; 14: 363-72.

Lingford-Hughes AR, Welch S, Nutt DJ (2004) Evidence-based guidelines for the pharmacological management of substance misuse, addiction and comorbidity: recommendations from the British Association for Psychopharmacology. Journal of Psychopharmacology; 18: 293-335.

Lintzeris N, Ritter A, Dunlop A (2002) Training primary health care professionals to provide buprenorphine and LAAM treatment. Substance Abuse; 23: 245-54.

Luty J, 0'Gara C, Sessay M (2005) Is methadone too dangerous for opiate addiction? The case for using a safer alternative, buprenorphine is strong. $B M J: 331: 1352-3$.

Magura S, Lee SJ, Salsitz EA, et al (2007) Outcomes of buprenorphine maintenance in office-based practice. Journal of Addiction Disorder; 26 : 13-23.

Marlatt GA, Gordon JR (1985) Relapse Prevention: A Self-Control Strategy for the Maintenance of Behaviour Change. Guilford Press.

Mattick RP, Kimber J, Breen C, et al (2002) Buprenorphine maintenance versus placebo or methadone maintenance for opioid dependence. Cochrane Database of Systematic Reviews; issue 4: CD002209.

Macleod J, Whittaker A, Robertson JR (1998) Changes in opiate treatment during attendance at a community drug service - findings from a clinical audit. Drug and Alcohol Review; 17: 19-25.

Mclntosh C, Ritson B (2001) Treating depression complicated by substance misuse. Advances in Psychiatric Treatment; 7: 357-64.

National Institute for Health and Clinical Excellence (2007) Drug Misuse: Opioid Detoxification. NICE (http://www.nice.org.uk/nicemedia/pdf/ CG52NICEGuideline.pdf).

O'Connor J, Moloney E, Travers R, et al (1988) Buprenorphine abuse among opiate addicts. British Journal of Addiction; 83: 1085-7.

Pradel V, Thirion X, Ronfle E, et al (2003) Assessment of doctor-shopping for high dosage buprenorphine maintenance treatment in a French region: development of a new method for prescription database. Pharmacoepidemiology and Drug Safety; 13: 473-81.

Raisch DW, Fudala PJ, Saxon AJ, et al (2003) Pharmacists' and technicians' perceptions and attitudes toward dispensing buprenorphine/naloxone to patients with opioid dependence. Journal of American Pharmacology Association; 45: 23-32.

Robertson JR, Raab GM, Bruce M, et al (2006) Addressing the efficacy of dihydrocodeine versus methadone as an alternative maintenance treatment for opiate dependence: a randomized controlled trial. Addiction; 101: 1752-9

Rosenheck R, Kosten T (2001) Buprenorphine for opiate addiction: potential economic impact. Drug and Alcohol Dependence; 63: 253-62.

Schifano F, Corkery J, Gilvan E, et al (2005) Buprenorphine mortality, seizures and prescription data in the UK, 1980-2002. Human Psychopharmacological Clinical Experiments; 20: 343-8.

Strang J, Sheridan J, Hunt C, et al (2005) The prescribing of methadone and other opioids to addicts: national survey of GPs in England and Wales. British Journal of General Practitioners; 55: 444-51.

Strang J, Manning V, Mayet S, et al (2007) Does prescribing for opiate addiction change after national guidelines? Methadone and buprenorphine prescribing to opiate addicts by general practitioners and hospital doctors in England, 1995-2005. Addiction; 102: 761-70.

Taikato M, Kidd B, Baldacchino A (2005) What every psychiatrist should know about buprenorphine in substance misuse. Psychiatric Bulletin; 29: 225-7.

Tracqui A, Kintz P, Ludes B (1998) Buprenorphine-related deaths among drug addicts in France: a report on 20 fatalities. Journal of Annalytical Toxicology: 22: 430-4.

Walsh SL, Preston KL, Stitzer ML, et al (1994) Clinical pharmacology of buprenorphine: ceiling effects at high doses. Clinical Pharmacology Therapy: 55: 569-80.

Winstock AR, Lea T, Ritter A (2007) The impact of community pharmacy dispensing fees on the introduction of buprenorphine-naloxone in Australia. Drug and Alcohol Review; 26: 411-6. 
Yanagita T, Kato S, Wakasa Y, et al (1982) Dependence potential of buprenorphine studied in rhesus monkeys. In Problems of Drug Dependence, 1981. National Institute on Drug Abuse Research Monograph 41)(ed LS Harris): 208-14. US Government Printing Office.
Zacny JP, Conley K, Galinkin J (1997) Comparing the subjective, psychomotor and physiological effects of intravenous buprenorphine and morphine in healthy volunteers. Journal of Pharmacology and Experimental Therapeutics; 282: 1187-97.

\section{MCQs}

1 NICE recommends that:

a only buprenorphine be offered as first-line treatment

b physicians should start detoxification with a different medication from that to be used for the maintenance treatment

c physicians can consider lofexidine for patients who are not suitable for methadone or buprenorphine

d physicians should consider lofexidine for elderly people with severe opioid dependence

e clonidine is routinely used in the treatment of opioid detoxification.

\section{The British Association for} Psychopharmacology recommends that:

a if short duration of treatment is desirable, methadone is preferable to $\alpha_{2}$-adrenergic agonists

b buprenorphine can be used for rapid withdrawal of opioids and has a better outcome than clonidine

c $\alpha_{2}$-adrenergic agonists are preferable to buprenorphine if there are concerns about bradycardia or hypotension

$\mathrm{d} \alpha_{2}$-adrenergic agonists result in lower severity of withdrawal symptoms than buprenorphine

e $\alpha_{2}$-adrenergic agonists are recommended to treat opioid withdrawal in pregnant women.

3 Regarding buprenorphine:

$\mathrm{a}$ it is an oripavine derivative of the opium alkaloid thebaine

b it is a partial $\kappa$-opioid agonist and $\mu$-opioid antagonist

c peak plasma concentration occurs approximately $10 \mathrm{~h}$ after dose administration

$d$ the low-dose formulation was licensed for the treatment of opioid addiction

e nausea, vomiting and constipation are rare side-effects.

4 Research shows that:

a in humans, buprenorphine does not produce euphoria and opioid-like effects

b there is absolutely no evidence to show that buprenorphine has reinforcing effects in individuals who are non-dependent and in recently detoxified opiate misusers

c sublingual preparations of buprenorphine are rarely injected intravenously by patients

$\mathrm{d}$ one variable that may contribute to the pattern of self-injection of buprenorphine is the presence of withdrawal symptoms

e buprenorphine has no undesirable withdrawal effects.

5 Regarding the global trend of buprenorphine prescribing:

a in the UK, $90 \%$ of doctors prescribe buprenorphine to their patients on a daily basis

b in the early 1990s, buprenorphine was one of the most common drugs of misuse by intravenous drug users in Scotland

c intravenous diversion of buprenorphine has not been recorded in France

$\mathrm{d}$ in the USA, physicians who prescribe buprenorphine are not required to possess certification in addiction medicine/psychiatry

e the prescription of buprenorphine is currently legal in Singapore. 